\title{
The Campus Digital-Book Reading and Communication Platform Based on Wifi and Android
}

\author{
HUI Xinning \\ Department of Computer Technology and Applications, \\ Qinghai University \\ Xining China \\ 1099748539@qq.com
}

\author{
LIU Yuli \\ Department of Computer Technology and Applications, \\ Qinghai University \\ Xining China \\ 912161136@qq.com
}

\begin{abstract}
In recent years the Android mobile terminal is popular in campus, at the same time the software of entertainment and making friends emerged one after another. However, there are only few platforms to study and communication for Android user in the numerous software. To solve the problem, we initiate the campus mobile digital-book reading and communication platform based on wifi, using Android application development and Java web technology, having the functions of online book-recommendation, book-reading, book-download, users-communication and so on. The platform is not only simple operation, low cost and strong practicability but also resolves the difficulty of seeking electronic books and communication with students in different areas.
\end{abstract}

Keywords-Android; campus communication platform; digital-book; reading

Android system uses the open-source Linux operating system, the underlying uses the $C$ language which is the fastest access to the hardware, the application layer uses a simple and powerful Java language which makes the Android in the mobile phone operating system particularly prominent[1].In this era of mobile Internet, Android system has unlimited potential.

In all kinds of colleges and universities, campus wireless network environment, combined with the characteristics of campus life and the popularity of the Android terminal prompted the needs of accessing to all kinds of electronic documents and books using a mobile phone anytime, anywhere. Therefore, the design of campus digital-book reading and communication platform based on wifi and Android has obvious practical significance.

The platform based on Android open-source operating system which was developed by Google company used the java web and wifi network environments. The system provides a large number of books which can be read online and be downloaded, and solves the current problems of finding books with difficulties and reading inconvenience. The system will create a good campus reading environment, and enhance students' interest in learning.

\section{SYSTEM ANALYSIS AND DESIGN}

1.1 System design

With the accelerated pace of life and the popularity of handheld devices, reading e-books have become an important way for people to increase their knowledge.
Reading software based on the Android platform are endless in recent years, but most of them are in favor of books reading of entertainment and they do not committ to learning and communication, and most of the software have the book format restrictions, failed to achieve unlimited format reading.

In addition, the mainly way of users accessing to electronic learning materials is through the network, but the network query is too wide, time-consuming and inconvenient to users .And book information display interface is unfriendly and a lot of ads affects the users' experience, most websites provide only a single reading and downloading. Instant communication can not be achieved between the user, and it can not create a good environment for learning with communications.

In this paper, we design a learning and communication platform based on Android. The system will provide a variety of e-books and the e-books will be classified when they were downloaded. The user can read e-books using the Android system software or the multifunction reader provided in the paper, in order to achieve unlimited format books reading.

The school users need to fill in the school name at the time of registration, in this way, the user can enjoy the pleasure of reading through the communication with the other users in the same school or downloaded the same books. Android attractive user interface elements promote users' enthusiasm to use and read. In addition, the system also automatically get the environment of the terminal equipment to achieve the adaptive screen display. The system interface displays more smoother.

1.2 System Framework

The system's client-side uses the SQLite database and the serve-side uses the open-source MySQL database. The server-side Servlet response HTTP request, the information that Servlet gets is sent to the client-side through the Dao interface which access background database, and returns the results.

In order to make the code easy to read, manage and secondarily develop, the system uses the MVC+DAO design pattern and hierarchical development thinking. The system's server-side is divided into receive and respond to requests module, as well as the back-end database storage module. The system's client-side is composed by online book information, reading module, communication module, and SQLite database storage module. Reading module and 
display module are the main functions of the system, and communication module is the major highlight.

\section{SYSTEM FUNCTIONS AND IMPLEMENTATION}

\subsection{Background MySQL database design}

The system's background MySQL database mainly has six data tables:book information table(bookinfo),book classification table(category),books download information table(download),book review table (comment),user message information table(leavemessage) and user information table(userinfo).Background server accesses the database via JDBC, the utility class DBUtil is used to open or close the database.The data model design is shown in Figure 2.

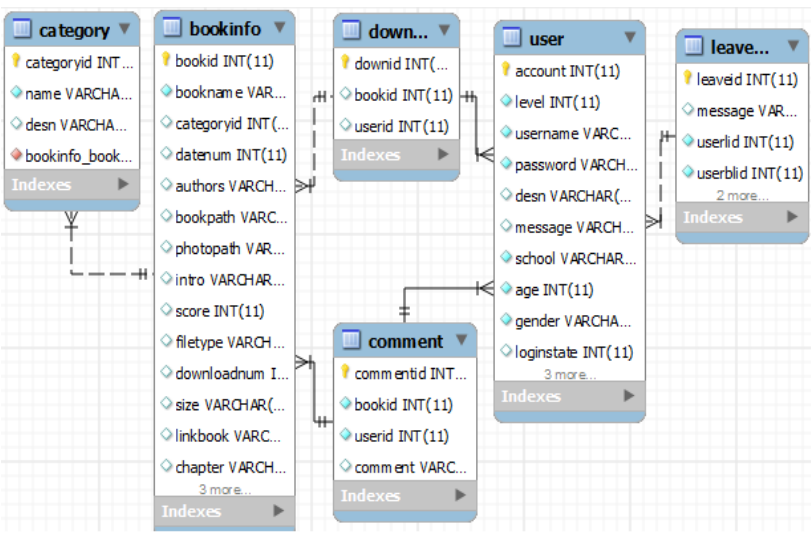

Figure 2 System Data Model

2.2 The basic functions of the client framework

The system achieves the functions of online books recommendation ,books classification, books searching, instant online reading, free ebooks download, users interaction and communication and so on, and we achieve unlimited format ebook reading using the Android system's software.System function framework is shown in Figure 3.

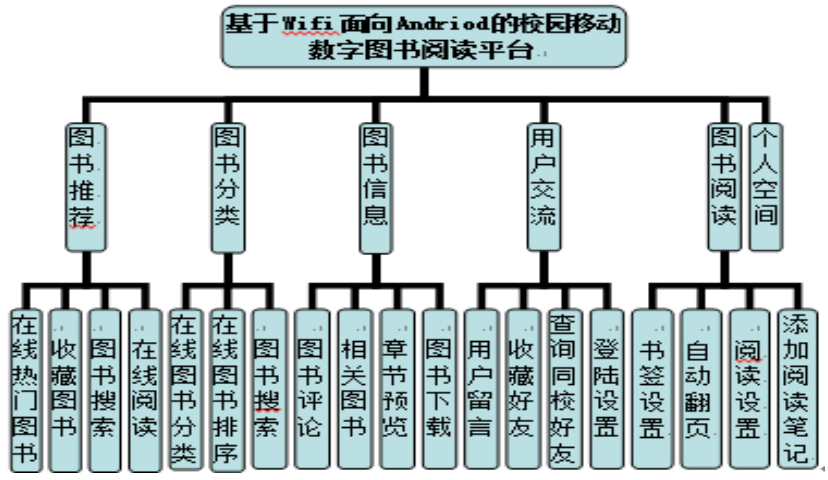

Figure 3 System Functional Framework Figure

\subsection{Reading module}

Reading module is divided into two parts: first, users can learn or read using the Android third-party software to open the downloaded ebooks, to achieve the unlimited format ebooks reading;Secondary,we use the multifunctional txt format book reader to read txt format ebooks and the reader implement many functions, for example, adding bookmarks, setting the background music, finding and reading books in the SD cards, setting the font size.In addition, the multifunctional txt format book reader achieved the dynamic flip books using Android animation[2], to provide users with a good reading experience. The key codes to open the e-book using the third-party software are as follows:

Intent intent $=$ new Intent () ;

intent.addFlags (Intent.FLAG_ACTIVITY_NEW_TASK);

/ /Set the intent of the Action Properties

intent.setAction (Intent.ACTION_VIEW);

/ /Get the MIME type of the file

String type = getMIMEType (file);

/ /Set the intent of the data and the Type attribute.

intent.setDataAndType (Uri.fromFile(file), type);

context.startActivity (intent);

/ /Jump to open the appropriate software

Downloaded books or reading books in the SD Card will mainly be classified as txt,pdf,doc and other format books through the book's format. In addition, the user can also add books into a list of my favorite books, therefore,it makes more convenient for users to manage the books.At present,the learning material mainly is divided into txt,pdf and doc format, so the system mainly provide reading and downloading based on the above three format ebooks, to enable the system to become a true learning platform, rather than an entertainment reading platform just for killing time.The read module is not just for reading but also for books category management.The main code for txt reader to draw text are as follows:

String txtstr =null;

/ / Read text

txtstr=TextLoadUtil.readFragment(start,

PAGE_LENGTH, FILE_PATH);

/ / the judgments text in Chinese or English

char $\mathrm{c}=$ txtstr.charAt (index);

//determine whether the text has finished

boolean isFinish = false;

int currRow $=0$; //line of text displayed

int currX $=0$;//Text drawing position

while(! isFinish)

$\{/ /$ To draw Chinese

canvas.drawText(c+"", currX + TEXT_SIZE/2, currRow*TEXT_SIZE + TEXT_SIZE, paint); currX $=$ currX+TEXT_SPACE_BETWEEN_CN;

The location to storage data for Android phone is not only the phone memory space but also includes a memory card(SD Card).Most of the works in the system's development and testing needs to be completed on the Android virtual machine, so it is necessary to create a memory card for the Android virtual machine. There are two ways to create a memory card in the virtual machine. The one way is to enter "mksdcard-1 sdcard $100 \mathrm{M}$ $\mathrm{d}$ :/ mycard.img" on the CMD command line window, it will create a memory card whose capacity is $100 \mathrm{M}$ and the position is 'd:/mycard.img'. The other way is to determine SD Card Size and set 'SD Card Support' to yes in Hardware 
on creating a virtual machine in Android Virtual Device Manager.

Downloaded or reading books mostly were stored in the SD card. In order to prevent the exception caused by SD card busying, the system will first detect the SD card state on reader module, and it will warn the users that books can not be read if the SD Card state is not normal. The key code to detect the status of the SD card are as follows:

String status =Environment.GetExternalStorageState ();

/ /Create StatFsobject

if(status.equal((Environment.MEDIA_MOUNTED))

// Determine the SD card status if normal return true

\{Return true;

2.4 Display Module

The system will get the Android network environment at runtime firstly, if network environment isn't wifi, the system will remind the user by the form of the dialog box, to avoid the loss of flow. We will ensure that the system is based on convenient and fast wifi through the detection and reminders of the network environment. The code to get the Android terminal wifi network environment are as follows:

WifiManager wifiManager;

wifiManager $=$ (WifiManager) this.getSystemService (Service. WIFI_SERVICE);

The display module contains online books recommendations, online book classification, query results, as well as detailed information about the books. In order to improve the user's experience, the system uses the multi-threaded and asynchronous way according to Android system thread-safe characteristics[3].The system will enters the wait interface while sending the request and waiting for a response results in the main thread(UI thread), in order to provide users with a better use experience.In the android platform, the new open thread can not access the Activity of Wight,therefore,it needs Handler mechanism for message delivery.At display module, the system sets child thread to complete the complex data crunching and deliver message through Handler mechanism to prevent the operation waiting too long in the main thread at the functions of books downloading or ebooks online reading[4].

The client request and the flow-chart is shown as figure 4.According to the user's operating request, the client response and open a child thread to send the request, background server receives the request and connect to MySQL database and returns the result of the request. Child threads get the results returned by the server and notify the main thread to make related reaction. The display of online books' information are mainly through the GridView in android.widget package.

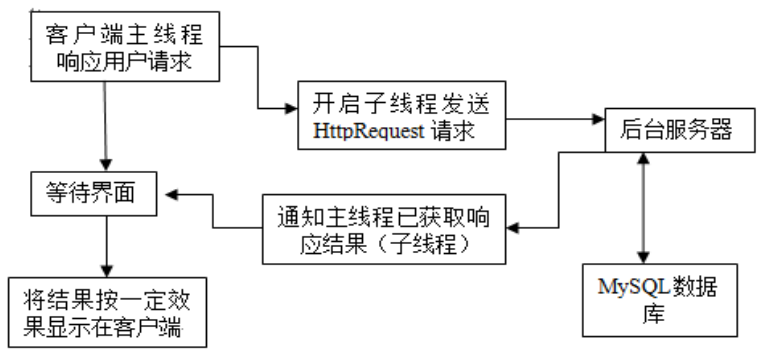

Figure 4 Client Requests and Display Flow-chart The key code of connection between Android client and the background server are as follows:

HttpPost request $=$ HttpUtil.GetHttpPost (url);

//UrlHttpPost object

String result $=$ null;

HttpResponse response

=HttpUtil.GetHttpResponse (request);

/ /Determine whether the request was successful if(response.getStatusLine (). getStatusCode () == 200) \{

result $=$ EntityUtils.toString(response.getEntity ()); return result; \}

\subsection{The data storage module}

Android achieves structured data storage by SQLite database engine. SQLite is an embedded database engine, and it provides an efficient database engine for limited memory resources devices (such as mobile phones, PAD, MP3)[5].The system's client use SQLite database to access data. The system design SQLiteUtil tools to perform various data manipulation. The system's client has eleven data tables, for example, reading books record table, bookmarks table, the last reading record table, favorite books table, downloaded books table and the messages table. The key code of the client's database operations to insert data to the reading record table are as follows.

createOrOpenDatabase() ;/ /open database

/ /Start the database query specified name exists

String sql = "select path from BookRecord where path = "' + path + "'"';

cur =sld.rawQuery (sql,new String [] \{\}$)$;

if(cur.moveToNext ())

$\{$ / /If it already exists, update sql $=$ "update BookRecord set data $=$ ? where path = "' + path + "'"; sld.execSQL (sql,new Object [] \{rdata\}); \}else

$\{/ /$ If there is inserted sql = "insert into BookRecord (path, data) values(?,?)"; sld.execSQL (sql, new Object [] \{path, rdata\}); 2.6 Communication module

Communication module is mainly used for the mutual exchange of registered users. It provides the functions such as leaving message, collecting user to the favorite friends list, finding the other users or the same school users and setting reading note.

If the user has logged and chosen to automatically receive a message in the account settings option, The system will prompt user to check the messages in the main interface of exchange when they have achieved some messages. In order to facilitate user learning and exchanging their mind with the others ,users can find the same school friends by the name of school filled in the user registration. The user can view the detailed information of the others, including the name, age and personal introduction. The user can also check books the others downloaded and look up who has downloaded the book. The communication module is 
important. It allows users to share their reading experience and makes the e-book reading no longer a closed and tedious place, so users can enjoy the pleasure of reading by the Android terminal.

\section{APPLICATION EFFECT}

The classification of the main interface of the online book is smooth,natural and user-friendly. It contains the function of screening books, searching books,sorting books and so on. Books classification divided into: computer, foreign language, cultural, scientific and others. The user can click on the appropriate category to screen the books, to facilitate the users to find what they want. Books Sort are divided into: by rating, by year and by downloads, to provide users with more choices to get the most wanted books. The overall operation of the effect is shown in Figure 5.

The users' communication interface running effect is shown in Figure 6.If the user has messages unread, the message control would prompt the unread message number. Click on the message control, the system will mark them as read. Users long-press will trigger onLongClickListener interface ,then we can delete or reply messages.

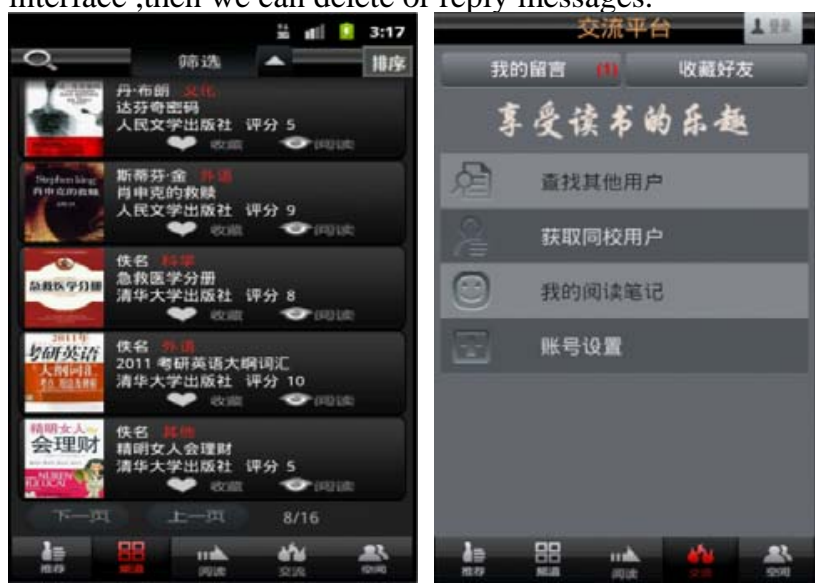

Figure 5 Book Classification Figure 6 User Communication

\section{CONCLUSION}

According to the development status of the mobile terminal and the needs of mobile users, we initiate the campus digital-book reading and communication platform based on wifi and Android. It has completed been tested, and put into operation. By simulation test on the virtual machine as well as actual testing on the Android phone terminal, the system is running stable and overall performing good. The system can provide mobile users with convenient, practical, flexible e-books learning and instant communication. With the popularity of smart phone and wifi network development, the system has a broad prospects.

\section{ACKNOWLEDGMENT}

This paper is funded by Education Support to Western Regions Program collaborated by Google and Tsinghua University.

\section{REFERENCES}

[1] Guo Hong-zhi.Android Application and Development Comments[M]. Beijing:Publishing House of Electronics Industry,2010.6

[2] Wu Ya-feng,Su Ya-guang. The Android Applications developed Daquan[M]. Beijing:People's Telecon Publishing House,2011.9

[3] ZHANG Cheng-jie .The Design and Realization of Online E-Book Microreader Using Android[J]. Microcomputer Applications,31.10 (2010):76-80

[4] WU Ya-feng,SUO Yi-na. The Android Core Technologies and Explain Examples[M]. Beijing:Publishing House of Electronics Industry, 2010.10

[5] HUANG Yi-feng,YAN Qiao.Design and Implementation of Electronic Dictionary Based on Android Platform[J]. Journal of Computer Applications,2011 31(2): 228 -232. 